\title{
A Survey on Current Underwater Acoustic Sensor Network Applications
}

\author{
Mohsin Murad, Adil A. Sheikh, Muhammad Asif Manzoor, Emad Felemban, and Saad Qaisar
}

\begin{abstract}
Terrestrial and airborne Wireless Sensor Networks rely on radio frequencies as their communication medium for transmitting data and information. However, sensing and subsequent transmission in sub-sea environment e.g. deep sea exploration requires all together a different approach for communication that has to be done under water. There's no escaping the fact that a huge amount of unexploited resources lies in the $70 \%$ of the earth covered by oceans. Yet, the aquatic world has mainly been unaffected by the recent advances in the area of Wireless Sensor Networks (WSNs) and their pervasive penetration in modern day research and industrial development. The current pace of research in the area of Underwater Acoustic Sensor Networks (UASNs) is at a snail's pace due to the difficulties arising in transferring most of the land and air based WSNs' state-of-the-art to their underwater equivalent. Maximum underwater deployments rely on acoustics for enabling communication combined with special sensors having the capacity to take on harsh environment of the oceans. This paper particularly focuses on gathering most recent developments and experimentation related to key underwater sensor network applications and UASNs deployments for monitoring and control of underwater domains.
\end{abstract}

Index Terms-Acoustic communication, acoustic sensor network, underwater sensor network, UASN.

\section{INTRODUCTION}

Underwater Acoustic Sensor Networks consists of a number of sensor nodes, stationary or mobile, connected wirelessly via acoustic communication modules deployed to monitor various events of interest collaboratively [1]. The objective is achieved by having a set of autonomous devices in a network which can self-organize and adapt to deep-sea conditions. Underwater communication is mainly done using

Manuscript received November 30, 2013; revised February 25, 2014. This work is supported by the NSTIP Strategic Technologies program number "11-INF1688-10", King Abdul Aziz City of Science and Technology of the Kingdom of Saudi Arabia. We thank Science and Technology Unit for their logistic support.

Mohsin Murad was with the Department of Computer Systems Engineering, University of Engineering and Technology, Peshawar, Pakistan and is with the Department of Computer Engineering and Simplicity Labs, Umm Al Qura University, Makkah, Saudi Arabia (e-mail: mmnkhan@uqu.edu.sa).

Adil A. Sheikh is with Science and Technology Unit and Simplicity Labs, Umm Al Qura University, Makkah, Saudi Arabia (e-mail: aasheikh @uqu.edu.sa).

Muhammad Asif is with the Department of Computer Engineering and Simplicity Labs, Umm Al Qura University, Makkah, Saudi Arabia (e-mail: mamanzoor@uqu.edu.sa).

Emad Felemban is with the Department of Computer Engineering, Umm Al Qura University and Simplicity Labs, Makkah, Saudi Arabia (e-mail: eafelemban@uqu.edu.sa).

Saad Qaiser is with National University of Science and Technology, Islamabad, Pakistan (e-mail: saad.qaisar@ seecs.edu.pk). low frequency and low data rate acoustic modems with a set of nodes transmitting their data to a buoyant gateway that relays the data to nearest costal monitoring and control station [2].

While UASN is a promising new field and may help in exploring what's hidden in the amazingly unfathomed world that lies underwater, there are a few challenges as well. For setting up underwater applications, not all the techniques and algorithms for UASNs can derive from the already seasoned land based WSNs as some of the phenomena are primarily different [3]. Radio frequency (RF) communications was never a choice for underwater communication due to the swift decrease in the transmission range and hence lower data rates of the RF modules as we go deep down the ocean. Acoustic communication has been the technology of choice for decades when it comes to transmitting signals underwater, but with their hefty price tags and heavy power usage, underwater acoustic modems are not at all feasible for UASNs with applications as itsy-bitsy as measuring the amount of pollution from a fishing farm at the sea bed [4]. Moreover, turning from speed of light to speed of sound changes the physics of communications incurring propagation delay and effects time synchronization. The sensors available are susceptible to routine underwater challenges e.g. algae collection on camera lens [5], salt accumulation; decreasing the affectivity of sensors etc. Finally, the energy requirements of UASNs will be different as compared to terrestrial WSNs for the fact that available underwater sensors have a larger foot print consuming higher power and regular battery replenishing techniques are quite costly. Very little work has been done in the field of underwater acoustic network deployments leaving the window wide open for upcoming research and opportunities.

In this paper, Section II discusses background of WSNs along with a short history of Underwater Sensing and UASNs. It is followed by literature review of selective research projects employing UASNs for a number of applications (Section III). In the end, various research opportunities in the domain of underwater sensor networks and final conclusion are discussed (Section IV).

\section{BACKGROUND}

The history of acoustic sensor networks dates back to simple ping based sonars that operated in the audible range. It was during the cold war era when a system of hydrophones was deployed on the seabed at different strategic areas to monitor the movement of soviet submarines. With the passage of time more sophisticated acoustic networks were developed for submarine and hostile ships detection. 
Ultrasonic communication has long been used in underwater vehicles for communication and has many similarities to RF but the only concern is the slow speed of sound underwater. The recent advancement in the areas of terrestrial WSNs and the availability of low cost sensors motivated the need for developing low cost applications and platforms to explore the oceans. Some low scale viable systems were available back in 1980s like the Datasonics modems in the US which operated on binary FSK having analog circuitry. Today there are a number of commercial acoustic modems as well as those built for in-house applications mainly funded by the industry or government [6] (see Fig. 1).

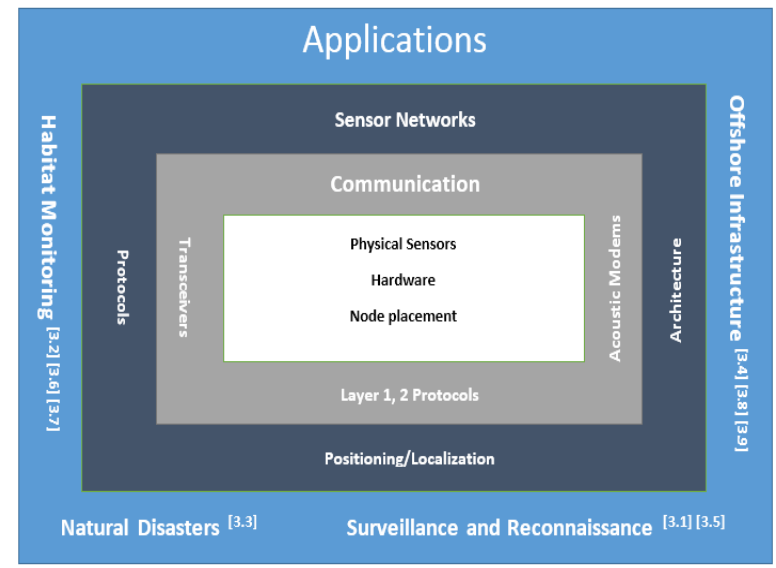

Fig. 1. Underwater acoustic sensor network architecture.

\section{UWSN APPLICATIONS}

Underwater sensor network applications based on acoustic communication are rapidly gaining popularity for enabling advances in the area of ocean monitoring and observatory systems, deep sea surveillance and tracking of various entities of the aquatic environment and unearthing resources. UASNs find their applications in fields like off shore oil and gas extraction, oil spills, military surveillance and reconnaissance, mine detection, pollution monitoring, natural calamities like tsunami and hurricane forecast, coral reef and habitat monitoring of marine life and fish farming to name a few. This section presents a survey of recent developments in the domain of UASN applications.

\section{A. Shallow Water Acoustic Network for Mine Countermeasures Operations [7]}

Lee Freitag, Matthew Grund et al. have presented an acoustic underwater network based on area mapping using gateway buoys and REMUS AUVs [8] to scan the area autonomously with very less human intervention. The paper explains deployment of an underwater acoustic network for communication and allowing the AUVs to map an area using its sonar and then classify potential targets. The network allows as seen in Fig. 2 for communication between multiple AUVs and they can exchange information with each other for an effective cooperative countermeasure operation. The acoustic modems used for communication are WHOI Micro-modems (Fig. 3) operating at about $25 \mathrm{kHz}$ and a bit rate of $80 \mathrm{bps}$ with the transducers mounted at the hull of the AUVs for maximum coverage. The gateway is equipped with RF and Acoustic communication modules to allow operation of the networked vehicles from a remote station. The gateway buoys have a Micro-Modem along with FreeWave RF modules in waterproof casing and buoyant by means of foam floatation. As the mission goes on, the operator at the control station receives real time data through the gateway buoy and is able to observe the number of mine like objects present in the survey area.

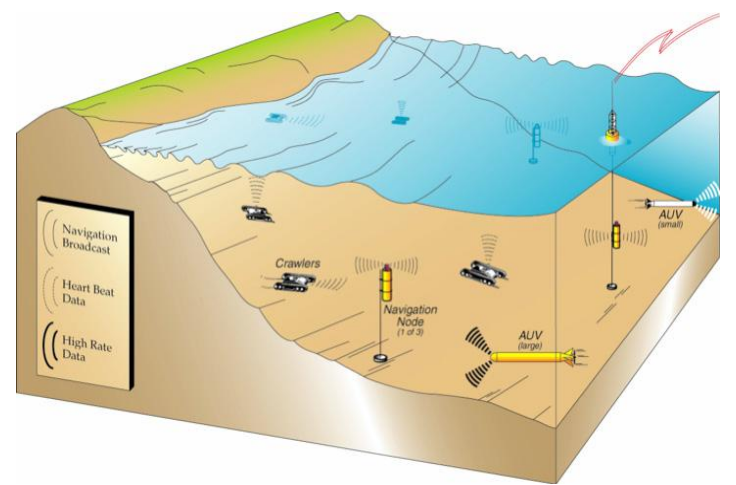

Fig. 2. Multiple vehicular mine counter measure system concept.

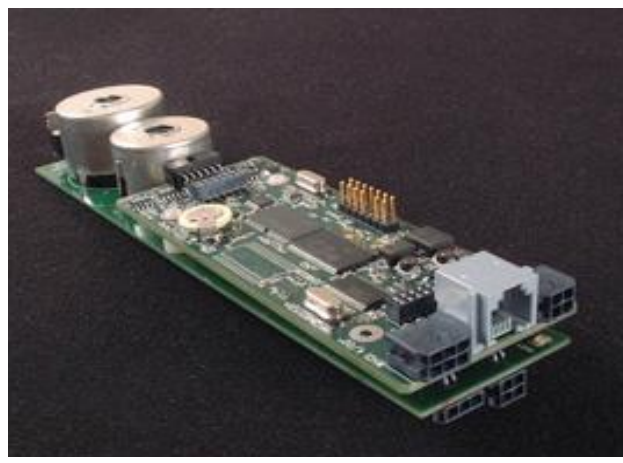

Fig. 3. WHOI micro-modem.

\section{B. Wireless Sensor Networks on the Great Barrier Reef}

Scott et al. present in [9] the lessons learned during the deployment of underwater sensor network hardware to study the effects of temperature, light intensity, salinity and other climatic conditions on coral bleaching. During the course of research the team emphasized on using time tested but expensive traditional underwater sensors rather than untested and inexpensive sensors, for instance a single sensor collapse can cause a huge loss in terms of vessel and workforce time. Also unlike land based WSNs, one cannot easily visit the location and change/service the faulty apparatus and therefore a single failure can incur costs as high as thousands of dollars.

Obtaining observatory data was rather an easy part but the main problem was finding the right people and research community who can utilize the data in a way that can help them in their ongoing researches. This was done through finding the right packages to integrate the outcomes with search engines and social networking platforms and make them intuitive. Biological fouling is a phenomenon in which anything under oceans will degrade either abruptly like in case of cyclones and tsunamis or slowly as in the case of algae formations with the passage of time. One has to plan well possible failures and the countermeasures taken to rectify those failures. One such method is to employ mechanical tools e.g. a wiper system (Zebra-tech: www.zebra-tech.co.nz/ hydro-wipers) that was used by the 
team (Fig. 4) to clean the light sensor by brushing anything that would try to settle over the sensor with the passage of time. However, as the amount of mechanical systems increase in a node, they will lessen battery lifespan. So far the system has effectively transmitted almost 2 million scientifically valid observations including some cyclones and a tsunami.

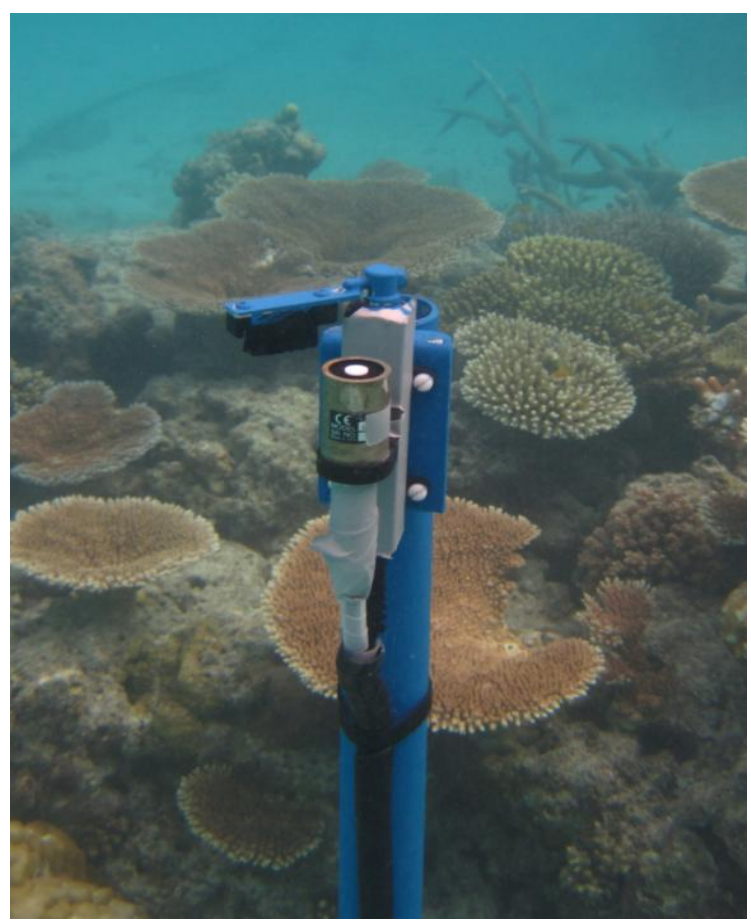

Fig. 4. Light meter with wiper on top to clean accumulating algae periodically [9].

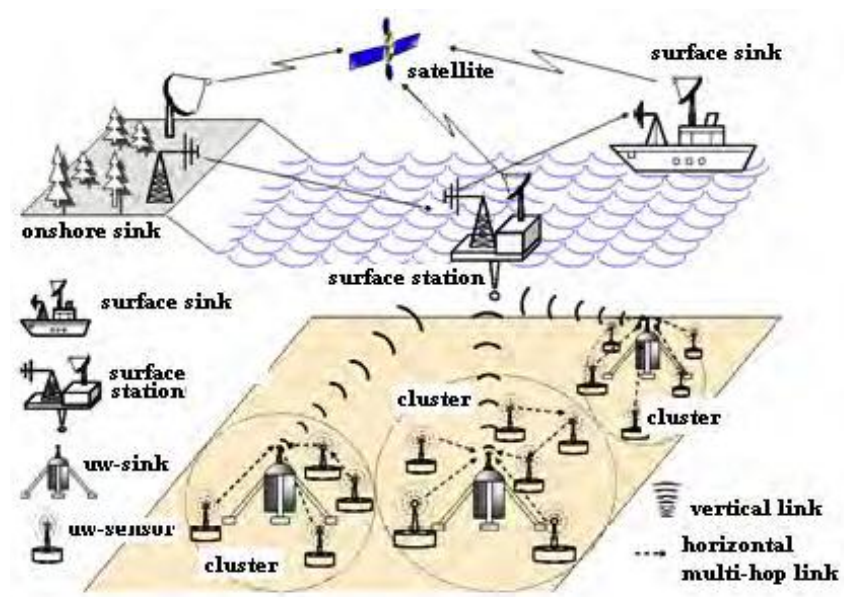

Fig. 5. Realization of an Early Warning System based on UWSN [10].

\section{UASN for Early Warning Generation of Natural Events}

The paper in [10], by Prashant et al., describes the physical layer challenges in design and development of a reliable, low power underwater wireless sensor network (UWSN) that can be globally utilized for early warnings against natural calamities like floods, tsunamis, earthquakes and hurricanes. Indicators such as seismic activity, variation in water turbulence, temperature and changes in the atmospheric densities can be measured by employing underwater sensor networks providing thorough information on any incoming disaster as early as a few days. The research proposes a three tier model having a number of wireless nodes deployed at the sea bed having acoustic modems for communication. The nodes are organized in groups and a group head accumulates the data and relays it to the nearest surface station. In the second level, microwave communication is used to relay the information collected at the surface node to the land based data collection center. Once the data reaches the coastal collection center, it can then be relayed to an international collection center via satellite communication (Fig. 5). Challenges that follow the model includes limited power, optimization of energy harvesting techniques, increased bit error rates (BER) and low signal to noise ratio (SNR) in case of low power nodes. Efficient routing techniques can play a vital role in controlling the power consumption by employing low power multi-hop transmission provided that the propagation delay do not exceeds the required limits.

\section{Sensor Network Architectures for Monitoring Underwater Pipelines}

In [11], Nader et al. have studied different sensor network models to monitor underwater pipelines. Their paper compares various possible architectures such as underwater wired sensor networks, UWSNs, wired/acoustic wireless sensor networks, RF based WSNs and wired/RF wireless networks that can be used to monitor any type of anomalies in a sub-sea pipeline. However, the literature that interests reader of this paper is the application of UWSNs for pipeline monitoring. An acoustic communication model (Fig. 6a) is used to measure disturbances due to vibrations in Langeled Pipeline below 1KM on an uneven and rocky seafloor where most parts of the pipeline aren't touching the seabed, hence highly susceptible to severe vibrations during strong currents.

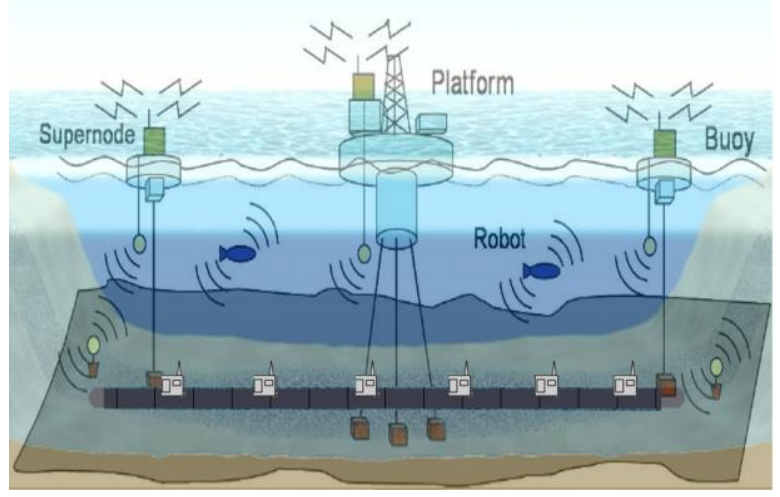

(a)

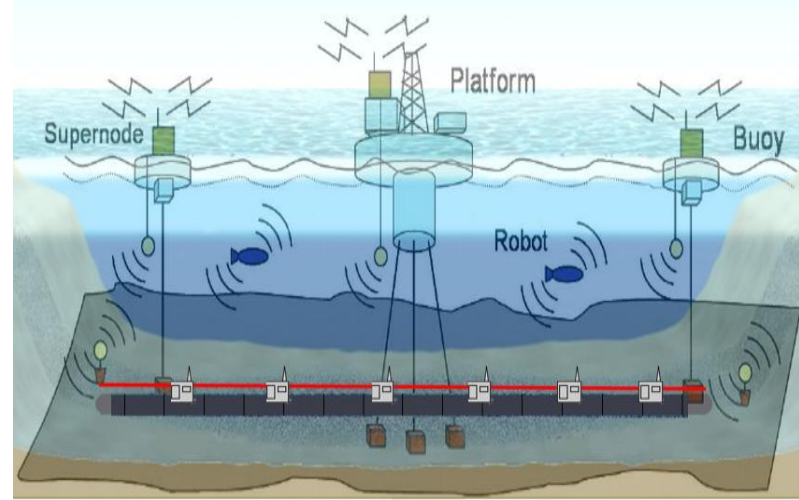

(b)

Fig. 6. (a) UWSN model for pipeline monitoring. (b) An integrated Wired/UWSN network. Adopted from Nader et al. ([11], Fig. 4). 
Dead nodes are replaced every 6 months after their batteries are worn out using a Remotely Operated Vehicle (ROV). The main limitation is the expensive upkeep and replacement jobs every 6 months. The proposed solution to this problem is integration of acoustic sensor nodes using a wired network (Fig. 6b). The power can be provided using the wired network architecture with each node having a rechargeable battery. Nodes can communicate both via wired or acoustic channels, in normal operation the wired channel is used, but in case of disruption or wire damage, the nodes will switch to their rechargeable batteries and acoustic communication to report the failure.

\section{E. Autonomous Underwater Surveillance Sensor Network (AUSSNet) [12]}

Engineers at L3 Oceania [13] have designed a sub-sea wireless network to be deployed in deep sea hostile territories of operational importance. AUSSNet can collect, process, store and forward critical surveillance data using acoustic modems to land, air, space or a nearby node/ship to enhance alertness and readiness to any unforeseen circumstances. The data is gathered at frequencies as low as $500 \mathrm{~Hz}$ and even less using acoustic line arrays that are positioned at the bottom of the sea in the area of operation. Once the data is received, it is processed for any dependent events and recorded on a permanent storage available in vicinity of that particular array. Later on, the data is transmitted to an access node that is installed on the seabed via underwater acoustic modems. The access node has a buoyant gateway (AGB) that it will release to the surface of the ocean automatically after preset intervals or when there is enough data and it relays the surveillance data to the nearby base station sub system for monitoring and control. Fig. 7 explains the overall architecture of the system with an Access Gateway Buoy (AGB), Control and Monitoring Station (CMS) and three underwater sensor nodes with acoustic modems.

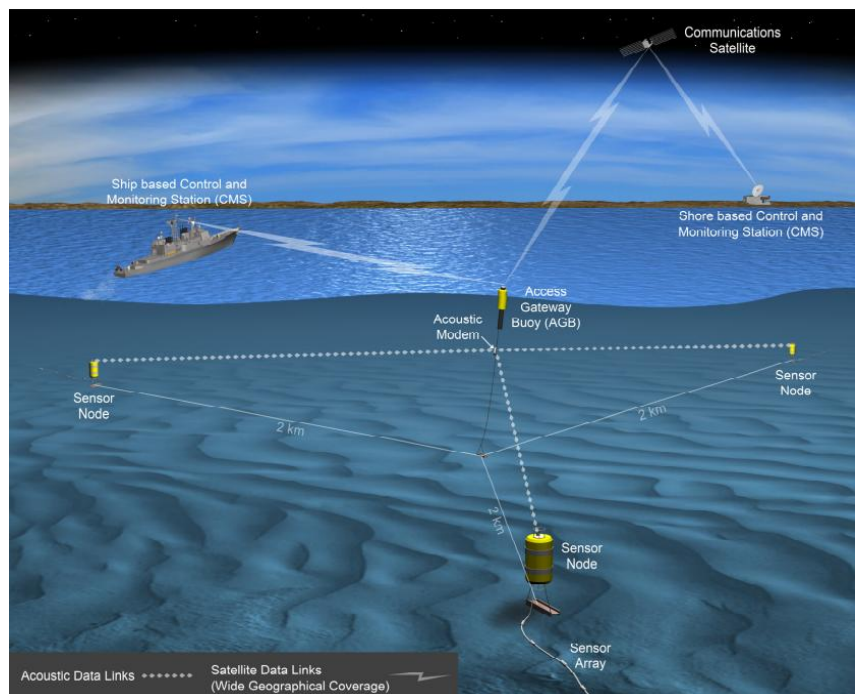

Fig. 7. Overview of the AUSSNet Architecture. Adopted from L3's Oceania AUSSNet Brochure [12].

\section{F. Group-based UWSN for Marine Fish Farms}

Jaime et al. [4] discuss a group based UWSN to measure the amount of pollution deposited at the sea floor because of the food not having been consumed and fecal remains in fish farms. An analytical model is used to find out the suitable location to place the underwater sensor nodes. The network can also be used to observe the quantity of fish feed that is wasted without being eaten as the nodes measure the amount of waste and left over reaching the sea bed. The proposed system can be very helpful in maintaining check and balance in countries where there are strict laws regarding the amount of waste generated from the marine farms.

UWSN can be handy in developing an effective monitoring system to observe the amount of waste generated as well as the amount of feed wasted. However, there are certain limitations such as the placement of the nodes exactly below the fish cages, the amount of nodes needed to cover the exact area and the sample period. Since a large amount of acoustic sensors nodes are required to cover the whole seabed exactly below the fish compartments, the authors propose using ultrasonic sensors placed $20 \mathrm{~cm}$ above the sea floor with their beams pointing towards the bottom to cover maximum area (Fig. 8)

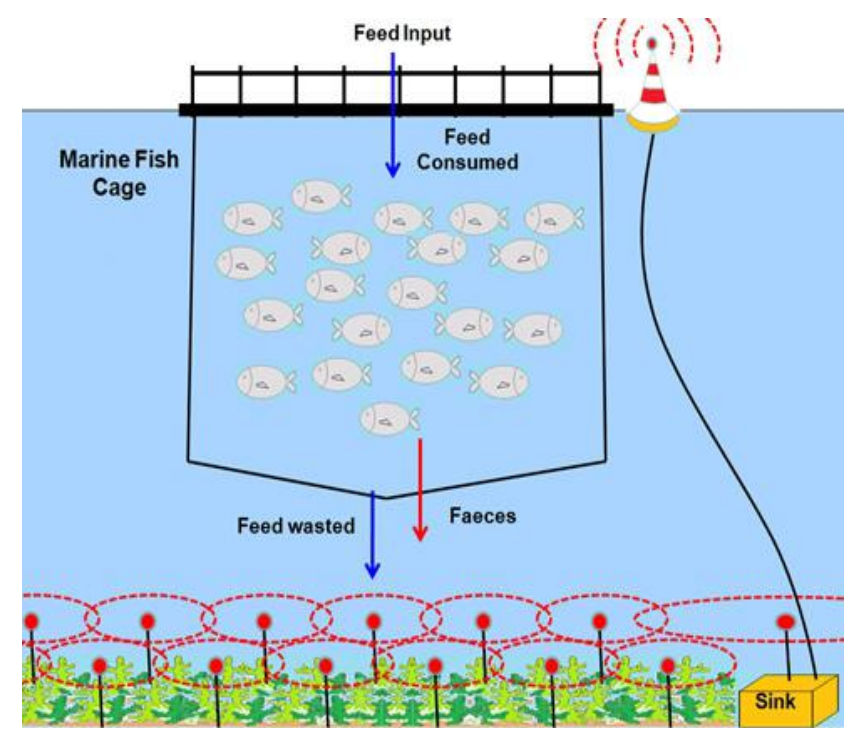

Fig. 8. UWSN for fish farm monitoring ([4]).

\section{G. UWSN for Water Quality Monitoring and Pollution Prevention}

Mustafa et al. in [14] propose an underwater sensor network to monitor the quality of water and the degree of pollutants. Underwater sensor nodes in their proposed test bench measure entities such as $\mathrm{pH}$, turbidity and temperature. The collected data is relayed to a node having GPRS connectivity, that will relay the information gathered to the nearest control center.

Authors in [15] have presented a decentralized ocean pollution and wreckage detection mechanism based on underwater sensor nodes equipped with short range acoustic modems for communication.

\section{H. Underwater Acoustic Network for the Protection of Offshore Platforms and Energy Plants}

The UAN [16] project under EU's 7th Framework Program aims to develop, design and test a working model based on underwater acoustic network equipped with multiple sensor systems to gather data vital in the monitoring 
of valuable offshore infrastructure to ensure its safety and security.

Such systems require a unified model based on aerial, ground and underwater sensor and actuator systems to guarantee foolproof protection.

The project targets at obtaining sensor data using the acoustic communication infrastructure. This data is also used to tune the communication system for optimal performance. The tuning process can be carried out by an Autonomous Underwater Vehicle (AUV) to physically separate the acoustic modules or bring them closer or it can work as a relay to far flung stationary nodes (Fig. 9).

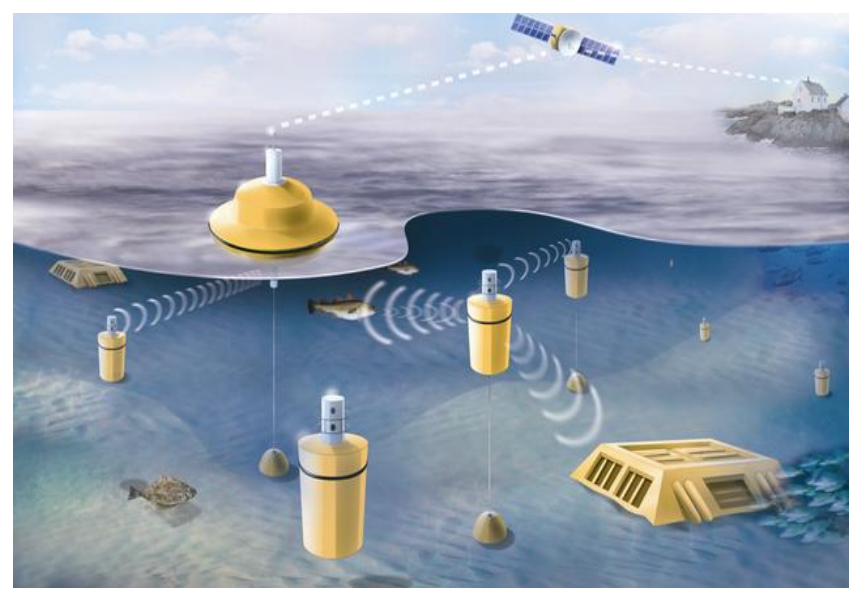

Fig. 9. UAN concept architecture [16].

\section{SeaSTAR Underwater Monitoring Platform}

Establishing an effective monitoring system aimed at underwater environment brings forth multiple challenges due to the limitation of communication options [17]. For effective sensing and monitoring a single independent unit has to coordinate with other neighbors in its proximity to perform its tasks. Due to limited data rates and short ranges, underwater communication was previously limited to simple controlling like shutting down underwater valves in an oil rig remotely or acoustically releasing a mooring. With the recent advances in technology it is now possible deploy a network of distributed nodes utilizing acoustic communication methods. The platform has applications of industrial, environmental monitoring, oil/gas exploitation, and safety and security.

\section{CONCLUSION}

In this paper, we have discussed a few most recent applications and field deployments of underwater acoustic sensor network. UASNs have a vast potential in exploring the dark yet interesting side of the oceans, provided that the pace of research continue to rise. Almost all of the applications that were brought to light in this effort, have agreed upon the fact that extensive research needs to be carried out in the area of acoustic communication to enhance the range and bandwidth of the underwater communication modems. With the advancement in the areas of sensing systems such as underwater visual sensor networks and real time streaming applications, we will require high bandwidth communication modules with lower latencies. Although these areas have seen a tremendous amount of growth in the past few years, there is still room for ample contributions particularly in physical network deployments.

\section{ACKNOWLEDGMENT}

This work is supported by the NSTIP strategic technologies program number "11-INF1688-10", King Abdul Aziz City of Science and Technology of the Kingdom of Saudi Arabia. We thank Science and Technology Unit for their logistic support

\section{REFERENCES}

[1] I. F. Akyildiz, D. Pompili, and T. Melodia, "Underwater acoustic sensor networks: Research challenges," Ad Hoc Networks, vol. 3, pp. 257-279, 2005.

[2] E. Felemban, "Advanced border intrusion detection and surveillance using wireless sensor network technology," Int. J. Communications Network and System Sciences, vol. 6, pp. 251-259, 2013.

[3] J. Heidemann, W. Ye, J. Wills , A. Syed, and Y. Li, "Research challenges and applications for underwater sensor networking," in Proc. IEEE Wireless Communications and Networking Conf., 2006.

[4] J. Lloret, S. Sendra, M. Garcia, and G. Lloret, "Group-based underwater wireless sensor network for marine fish farms," presented at IEEE GLOBECOM Workshops, 2011.

[5] J. Mullica, J. Krisanadej, B. Scott, F. Tony et al., "Sensor Networks Applications for Reefs at Racha Island, Thailand," in Proc. 12th International Coral Reef Symposium, 2012

[6] M. Chitre and S. Shahabudeen, "Recent advances in underwater acoustic communications \& networking," in Proc. IEEE Oceans, 2008.

[7] L. Freitag, M. Grund, C. von Alt, R. Stokey, and T. Austin, "A shallow water acoustic network for mine countermeasures operations with autonomous underwater vehicles," presented at the IEEE Oceans Conference, Washington DC, 2005.

[8] C. von Alt, B. Allen, T. Austin, and R. Stokey, "Remote environmental measuring units," presented at Autonomous Underwater Vehicle Conference '94, Cambridge, MA., 1994.

[9] B. Scott, E. Damien, and G. Page, "Lessons from the field-Two years of deploying operational wireless sensor networks on the great barrier reef," Sensors, vol. 11, pp. 6842-6855, 2011.

[10] P. Kumar, P. Priyadarshini et al., "Underwater acoustic sensor network for early warning generation," in Proc. IEEE Oceans, 2012.

[11] M. Nader, J. Imad, J. Al-Jaroodi, and Z. Liren, "Sensor network architectures for monitoring underwater pipelines," Sensors, vol. 11, pp. 10738-10764, 2011.

[12] AUSSNet - Autonomous Underwater Surveillance Sensor Network. [Online]. Available: http://www2.1-3com.com/oceania//products/ maritime_aussnet.htm

[13] Oceania. [Online]. Available: http://www2.1-3com.com/oceania/

[14] M. Shakir, M. A. Khan, S. A. Malik, and Izhar-ul-Haq, "Design of underwater sensor networks for water quality monitoring," World Applied Sciences Journal, vol. 17, no. 11, pp. 1441-1444, 2012.

[15] A. Khan and L. Jenkins. "Undersea wireless sensor network for ocean pollution prevention," in Proc. 3rd International Conference on Communication Systems Software and Middleware and Workshops, 2008.

[16] Project UAN. [Online]. Available: http://www.ua-net.eu/

[17] SeaSTAR (ASSYS). [Online]. Available:http://www.utwente.nl/ctit/ research/projects/national/stw/seastar.doc/

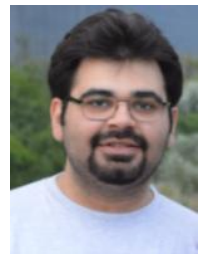

Mohsin Murad completed his masters in computer systems engineering from University of Engineering and Technology, Peshawar, Pakistan in 2012. Earlier he finished bachelors in computer systems engineering with honors from the aforementioned university in 2009.

Currently, he is working as a lecturer in the Department of Computer Engineering at Umm Al Qura University, Makkah, Saudi Arabia since 2012. Before joining Umm Al Qura University, he was employed as a researcher in a Public Sector Organization for the Government of Pakistan. He has also served as a lecturer in the Department of Computer Systems Engineering, University of Engineering and Technology, Peshawar, Pakistan. His research interests include applications of wireless sensor networks, networked embedded systems, cyber physical systems, RFID and real time tracking. 


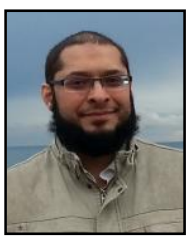

Adil A. Sheikh finished his double masters through the Erasmus Mundus in 2007. He majored in embedded systems from University of Trento, Italy and RWTH-Aachen, Germany. Earlier he completed his BE from National University of Sciences and Technology, Pakistan with Rectors Gold Medal, in 2001.

Currently, he is working as a researcher in the Science and Technology Unit, Umm Al-Qura University, Saudi Arabia. His research interests include visual sensor networks, RFID applications, intelligent transportation systems and e-health projects.

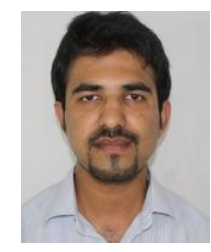

Muhammad Asif Manzoor obtained his M.Sc. and B.Sc. degrees from the Department of Computer System Engineering at the University of Engineering and Technology, Peshawar, Pakistan in 2007 and 2010 respectively.

Currently he is working as a lecturer at the Computer Engineering Department at Umm Al-Qura University, Saudi Arabia. He is currently working in multiple research areas including visual sensor networks, embedded systems, image processing and cyber physical systems.

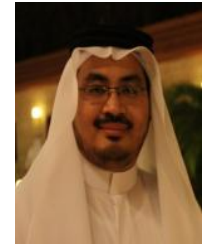

Emad Felemban earned his M.Sc. and $\mathrm{PhD}$ from Ohio State University in 2003 and 2009, repetitively.

$\mathrm{He}$ is working as an assistant professor in the Computer Engineering Department Umm Al-Qura University, Makkah, Saudi Arabia. He is also leading the transportation and crowd management center of research excellence in Umm Al-Qura University working on cutting edge research. His research interests include wireless sensor networks algorithm and protocols, smartcity applications, and smart antennas.

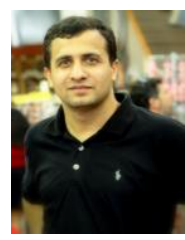

Saad Qaiser received his masters and Ph.D. degrees in electrical engineering from Michigan State University, USA in 2005 and 2009, respectively, under supervision of Dr. Hayder Radha, who is a fellow of IEEE.

$\mathrm{He}$ is currently serving as an assistant professor at School of Electrical Engineering \& Computer Science, National University of Sciences \& Technology (NUST), Pakistan. He is the lead researcher and founding director of CoNNekT Lab: Research Laboratory of Communications, Networks and Multimedia at NUST. He has published over 20 papers at reputed international venues with a vast amount of work in pipeline. 